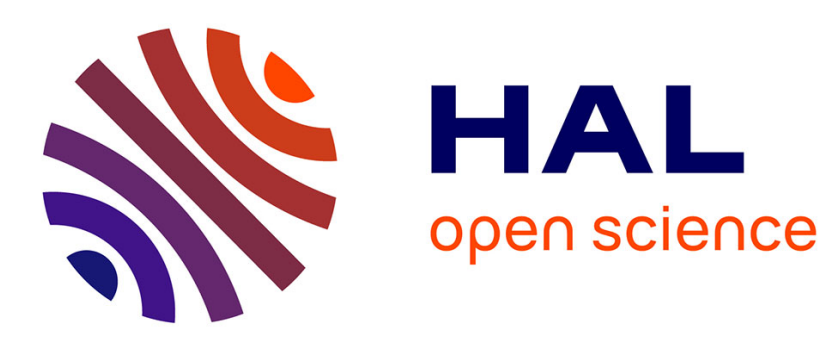

\title{
Surface geometry and local critical behaviour : the self-avoiding-walk
}

Loïc Turban, Bertrand Berche

\section{To cite this version:}

Loïc Turban, Bertrand Berche. Surface geometry and local critical behaviour : the self-avoiding-walk. Journal de Physique I, 1993, 3 (4), pp.925-934. 10.1051/jp1:1993173 . jpa-00246773

\section{HAL Id: jpa-00246773 https://hal.science/jpa-00246773}

Submitted on 1 Jan 1993

HAL is a multi-disciplinary open access archive for the deposit and dissemination of scientific research documents, whether they are published or not. The documents may come from teaching and research institutions in France or abroad, or from public or private research centers.
L'archive ouverte pluridisciplinaire HAL, est destinée au dépôt et à la diffusion de documents scientifiques de niveau recherche, publiés ou non, émanant des établissements d'enseignement et de recherche français ou étrangers, des laboratoires publics ou privés. 
Classification

Physics Abstracts

$05.50-64.60 \mathrm{~F}-36.20 \mathrm{C}$

\title{
Surface geometry and local critical behaviour : the self- avoiding-walk
}

\author{
Loï Turban and Bertrand Berche \\ Laboratoire de Physique du Solide (*), Université de Nancy I, BP 239, F-54506 Vandœuvre-lès- \\ Nancy Cedex, France
}

(Received 21 October 1992, accepted 27 November 1992)

\begin{abstract}
The statistics of a polymer chain confined inside a system which is limited by a parabolic-like surface $v= \pm C u^{k}$ is studied through Monte-Carlo simulations in two dimensions. In agreement with scaling considerations, the surface geometry is found to be a relevant perturbation to the flat surface behaviour when the shape exponent $k$ is smaller than one. In this case the system becomes anisotropic with a radius exponent $\nu^{p}$ along the parabola greater than the exponent $\nu_{\perp}^{p}$ in the transverse direction. When $k<1$ the anisotropy ratio $z$ adjusts itself to the value $k^{-1}$ for which the surface geometry is a marginal perturbation. The exponents obtained analytically, using either the blob picture approach or a Flory approximation, are in good agreement with the $2 \mathrm{~d}$ simulation results.
\end{abstract}

\section{Introduction.}

Isotropic systems confined in corners, wedges or cones are known to display marginal local critical behaviour, the local magnetic exponent varying continuously with the opening angle. This result, first obtained by Cardy [1] in mean field and through an $\varepsilon$-expansion near $d_{\mathrm{c}}=4$ in the $\varphi^{4}$-theory, has been since then implemented by exact results in $2 \mathrm{~d}$ using conformal techniques [2-3]. The Ising corner magnetization has also been deduced from startriangle recursion equations [4], from the corner-corner correlation function [4-6] or using the corner transfer matrix [7]. Some exact results have been obtained for the self-avoiding walk (SAW) [3, 8]. Marginal behaviour may be traced to the absence of a length scale in such geometries. The opening angle, which is invariant in an isotropic change of scale, may be identified as the marginal variable.

More recently, the case of a "parabolic » surface geometry was considered [9]. The system is then limited by a parabola

$$
v= \pm C u^{k}
$$

(*) URA CNRS n 155 . 
in $2 \mathrm{~d}$ (Fig. 1), a paraboloid or a parabolic wedge in 3d. In this geometry $C^{-1}$ plays the same role as $L^{-1}$ in finite-size scaling and can be considered as a scaling variable associated with the perturbation introduced by the curved surface with respect to the flat surface fixed point for which $C^{-1}=0$. Under an isotropic change of the length scale by a factor $b$, it transforms according to

$$
C^{\prime-1}=b^{1-k} C^{-1}
$$

so that the perturbation is irrelevant when $k>1$, marginal when $k=1$ and relevant when $k<1$. The case $k=0$ corresponds to a strip geometry in $2 \mathrm{~d}$, a slab or a tube in $3 \mathrm{~d}$. In the marginal case, $k=1$, one recovers the corner, wedge and cone geometries mentioned above. In $2 \mathrm{~d}$, a conformal mapping of the semi-infinite system was used in [9] to show that, for a relevant perturbation, the local correlations at the bulk critical point display a streched exponential behaviour which may be considered as a generalization of the well-known finitesize scaling result for the strip correlation functions. Furthermore, using scaling arguments or a corner transfer matrix method in the Hamiltonian limit, the Ising tip magnetization was shown to vanish with an essential singularity at the bulk critical point.

In this paper we study the critical behaviour of the SAW confined inside a parabola with one of its end points fixed near to the tip. This walk is known to describe the statistics of a polymer chain in a good solvent [10]. Although the $2 \mathrm{~d}$ behaviour is mainly considered, most of the conclusions still apply in $3 \mathrm{~d}$. Section 2 is devoted to a scaling analysis of the behaviour of the chain radii, $R_{\sharp}$ along the axis of the parabola and $R_{\perp}$ in the transverse direction. In section 3 the results of a $2 \mathrm{~d}$ Monte-Carlo simulation are presented. In the next section we show how the chain conformation can be understood using either the blob picture [10-11] or a Flory approximation. In the final section, we discuss our results and show how they may be extended to other isotropic polymer problems as well as to systems displaying from the start an anisotropic critical behaviour.

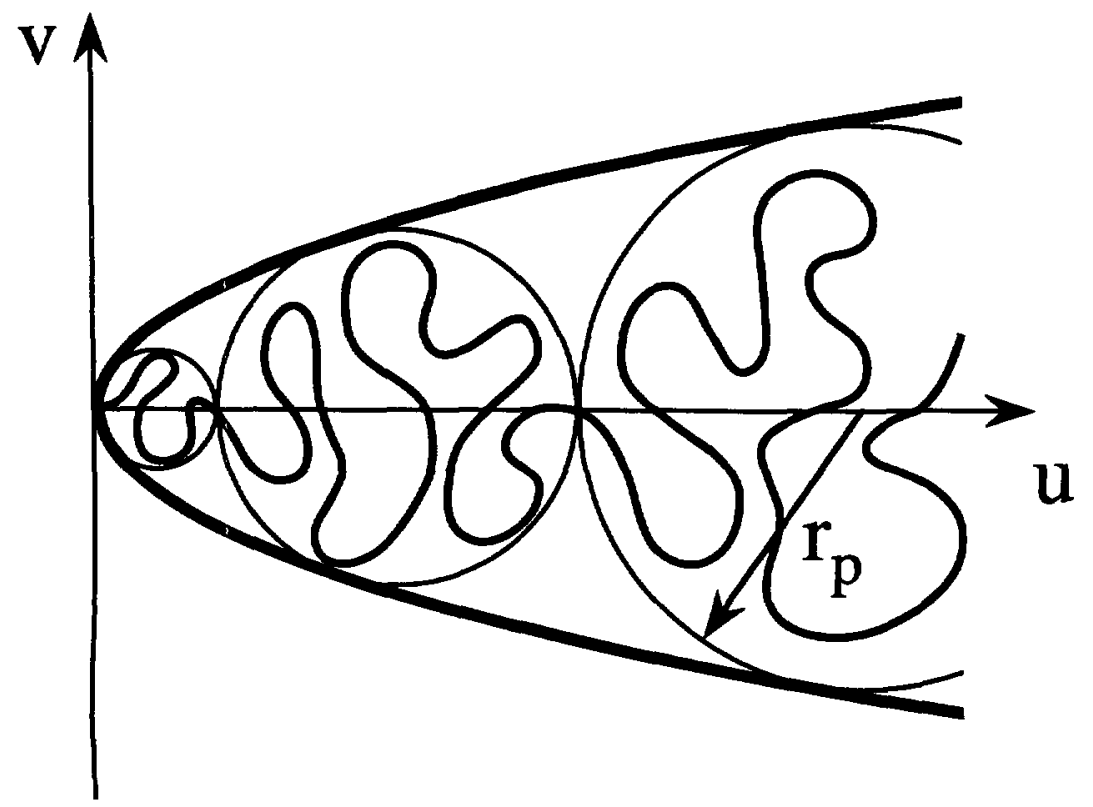

Fig. 1. - Parabolic geometry considered in the text. The confined polymer chain configuration results from the piling of fractal blobs inside the parabolá. 


\section{Scaling considerations.}

We consider a chain with $N$ monomers ( $N$-step SAW) confined into a system limited by a parabolic surface. Equation (1) either gives the surface itself in $2 d$ or corresponds to a section of the system in 3d. Due to the constraint imposed by the surface, the chain radii will behave in different ways along the axis $\left(R_{\|}\right)$and in the transverse direction $\left(R_{\perp}\right)$. In $3 \mathrm{~d}$, for a wedge, the behaviour would be still different in the third direction, perpendicular to the $(u, v)$ section. Under an isotropic change of scale, the root-mean-square radii transform according to

$$
R_{\|, \perp}(N, C)=b R_{\|, \perp}\left(b^{-1 / \nu} N, b^{k-1} C\right)
$$

where the reference fixed point is the flat surface one with the correlation length exponent $\nu$ taking its bulk value. With $b=N^{\nu}$ one gets

$$
R_{1, \perp}(N, C)=N^{\nu} R_{\|, \perp}\left(1, N^{\nu(1-k)} C\right) .
$$

Introducing the length scale

$$
R_{C} \sim C^{1 /(1-k)}
$$

associated with the parabolic geometry, one may rewrite (4) as

$$
R_{\|, \perp}(N, C)=N^{\nu} f_{\|, \perp}\left(R_{C} / N^{\nu}\right)
$$

where $N^{\nu} \sim R(N)$, the chain radius in the flat surface geometry. The $k$-dependent scaling function $f_{\|, \perp}(x)$ describes the crossover from the flat surface behaviour in the limit $N^{\nu} \ll R_{C}$ where it goes to a constant, to the parabolic behaviour in the limit $N^{\nu} \gg \dot{R}_{C}$. In the latter case, since a new fixed point with its own exponents is involved, a power law behaviour in the scaled variable $x$ is expected

$$
f_{\|, \perp}(x) \sim x^{\omega_{\|, \perp}} \quad x \rightarrow 0
$$

leading to a new set of local exponents

$$
\nu \mathbb{P}_{, \perp}=\nu\left(1-\omega_{\|, \perp}\right)
$$

for the $N$-dependence of $R_{\|}$and $R_{\perp}$. Due to the geometrical constraint, $R_{\perp}$ scales with $N$ like $R_{\|}^{k}$ i.e. $\nu_{\perp}^{p}=k \nu P$ and the exponents giving the small- $x$ behaviour of the two scaling functions have to satisfy the scaling relation

$$
\omega_{\perp}=1+k\left(\omega_{\|}-1\right) .
$$

\section{Monte-Carlo simulations in two dimensions.}

The 2d SAW problem has been studied numerically using Monte-Carlo techniques [12]. In order to face the difficulties associated with systems displaying crossover effects, long chains and a good statistics are needed. We used an efficient unbiased method combining simple sampling [13], the "pivot » algorithm [14] and the enrichment method [15].

We worked on a square lattice with coordination number $q=4$. First, a self-avoiding chain with $N_{1}$ steps, starting near to the tip of the parabola, is generated through the simple sampling method. At each step one of the $q-1$ directions avoiding a direct return is selected at random. 
If the chain reaches an occupied site or leaves the parabola, a new chain is started. When the chain with $N_{1}$ monomers is obtained, the «pivot» algorithm, which has been proved to be ergodic [16], is used to generate new configurations. One of the occupied sites is randomly selected (Fig. 2a) and the end part of the chain is rotated at random around it (Fig. 2b). The new configuration is accepted when it is self-avoiding and remains inside the parabola. Otherwise the old configuration is counted once more. This process is repeated until the required number of configurations is obtained. The chain is then enriched by $N_{1}$ steps through the simple sampling method, starting with the last configuration given by the "pivot " algorithm. When $N_{1}$ steps are successful, new configurations for the longer chain are generated through the «pivot» algorithm and the whole sequence is repeated until the maximum chain size is reached.

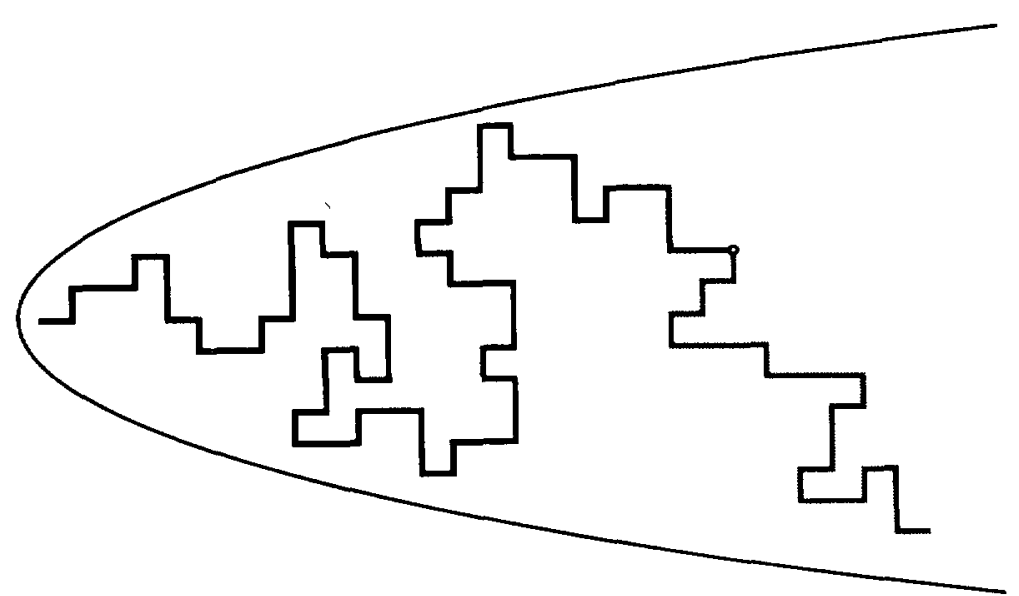

a)

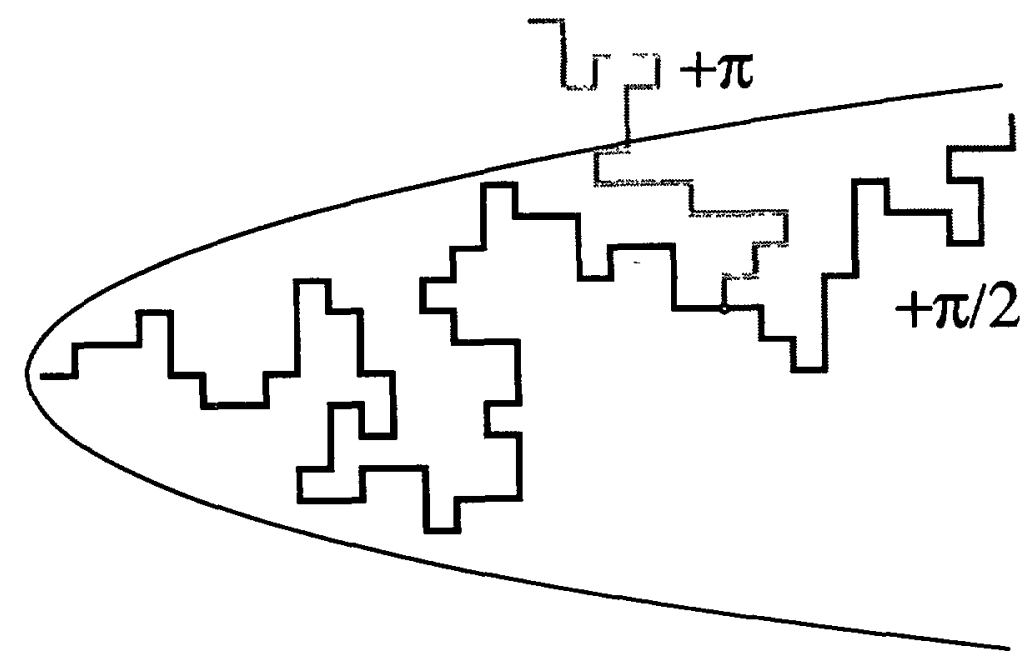

b)

Fig. 2. - Pivot algorithm : (a) random selection of the pivot on the original configuration; (b) construction of the new configuration through random rotation (here only the $+\pi / 2$ rotation is acceptable). 
SAWs of up to 200 steps were generated in this way for different values of $C(2,2.5,3)$ and $k(1 / 4,1 / 3,1 / 2,2 / 3,1,2)$. The square of the end-to-end radii, $R\}$ and $R_{\perp}^{2}$, were averaged over a number of samples going from $2-3 \times 10^{5}$ for $k=2$ to $3-4 \times 10^{6}$ for $k=1 / 4$. Although the radius of gyration is less fluctuating than the end-to-end radius, it leads to a slower crossover to the asymptotic behaviour when the walk length is increased. This is why the second was preferred.

Log-log plots of the scaling functions $f_{\|, \perp}(x)$ are shown in figure 3 for $k=1 / 2$. The data collapse on a single curve for the different values of $C$. The asymptotic slopes give the scaling function exponents $\omega_{\|, \perp}$ defined in equation (7). Finite-size estimators for the end-to-end radius exponents were obtained using

$$
\nu \mathbb{R}_{, \perp}(N)=\frac{1}{2} \frac{\ln \left[\overline{R_{, \perp}^{2}}(N+i)\right]-\ln \left[\overline{R_{, \perp}^{2}}(N-i)\right]}{\ln (N+i)-\ln (N-i)}
$$

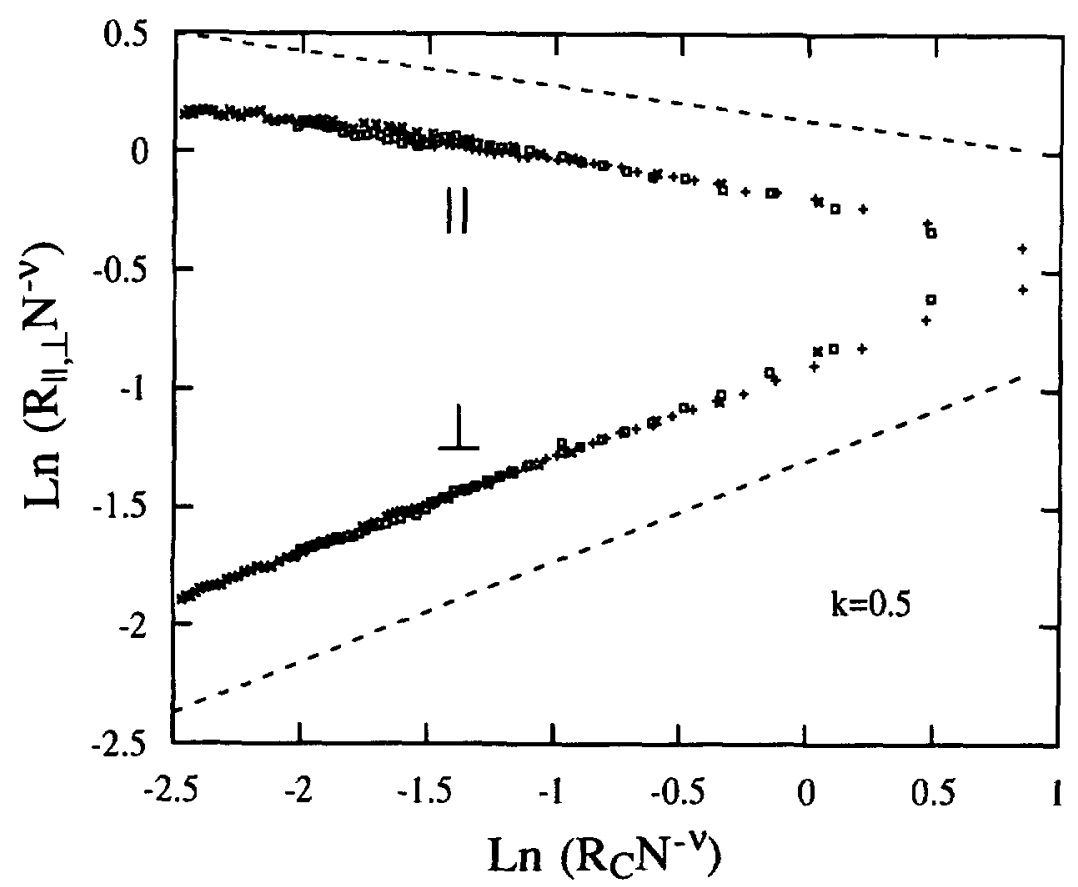

Fig. 3. - Log-log plot of the end-to-end radius scaling functions for $k=0.5$ and different values of $C((\times) 2,(\square) 2.5,(+) 3)$. The dashed lines give the theoretical asymptotic slopes.

In order to reduce the statistical noise an average was taken over the $i+1$ successive estimators around $N$. The averaged estimators are plotted in figure 4 as functions of $N^{-1}$ for $k=1 / 2$ and different values of $C$. Since the resulting curves are practically horizontal, the final estimates of $\nu p_{\perp}$ were obtained by averaging over these estimators, the error corresponding to one standard deviation. The $k$-dependence is shown in figure 5 for $C=3$. Within the data accuracy the results are the same for $C=2$ and 2.5. When the parabolic shape is a relevant perturbation, i.e. for $k<1$, the critical behaviour becomes anisotropic with $\nu \mathbb{P}>\nu_{\perp}^{p}$ and both exponents are $k$-dependent. 


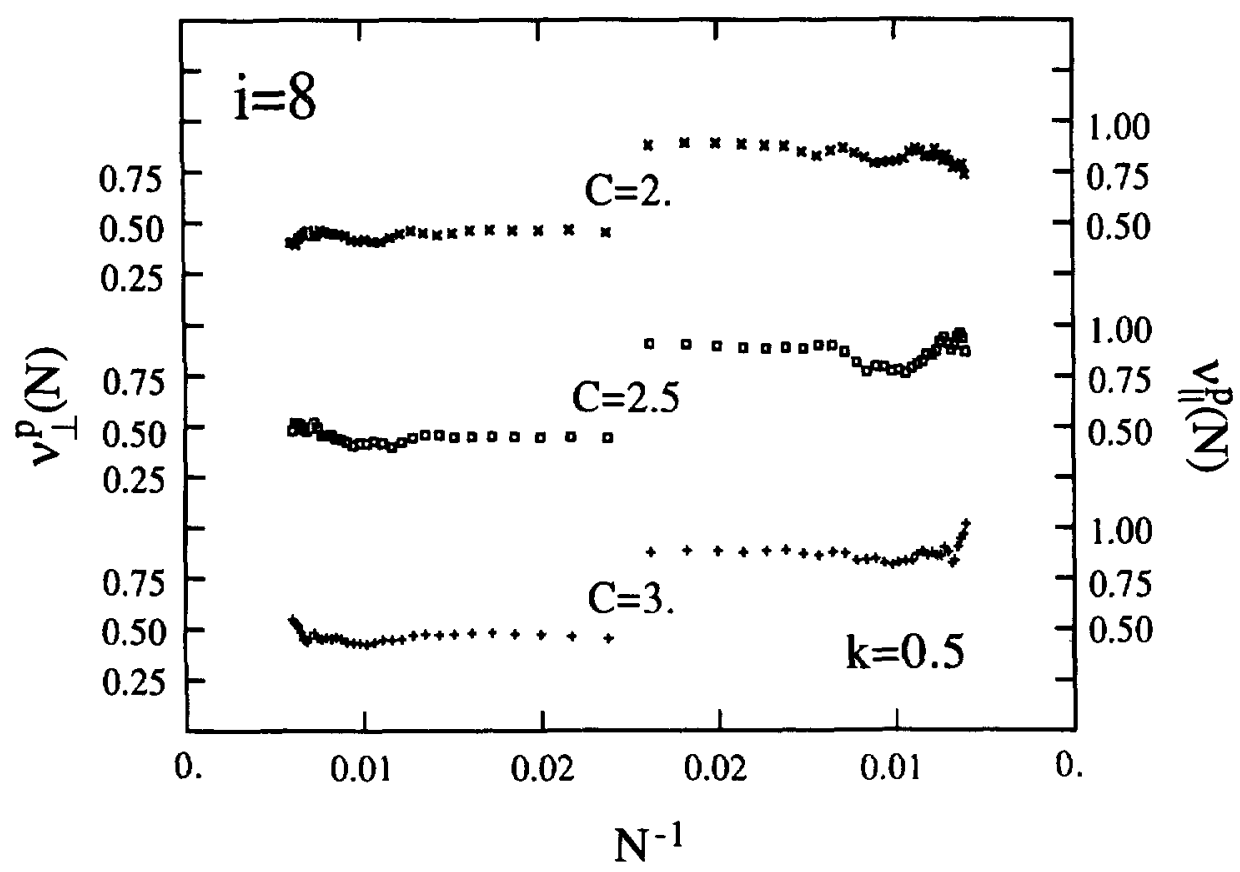

Fig. 4. - Estimates of the radius exponents as functions of $N^{-1}$ for $k=0.5$.

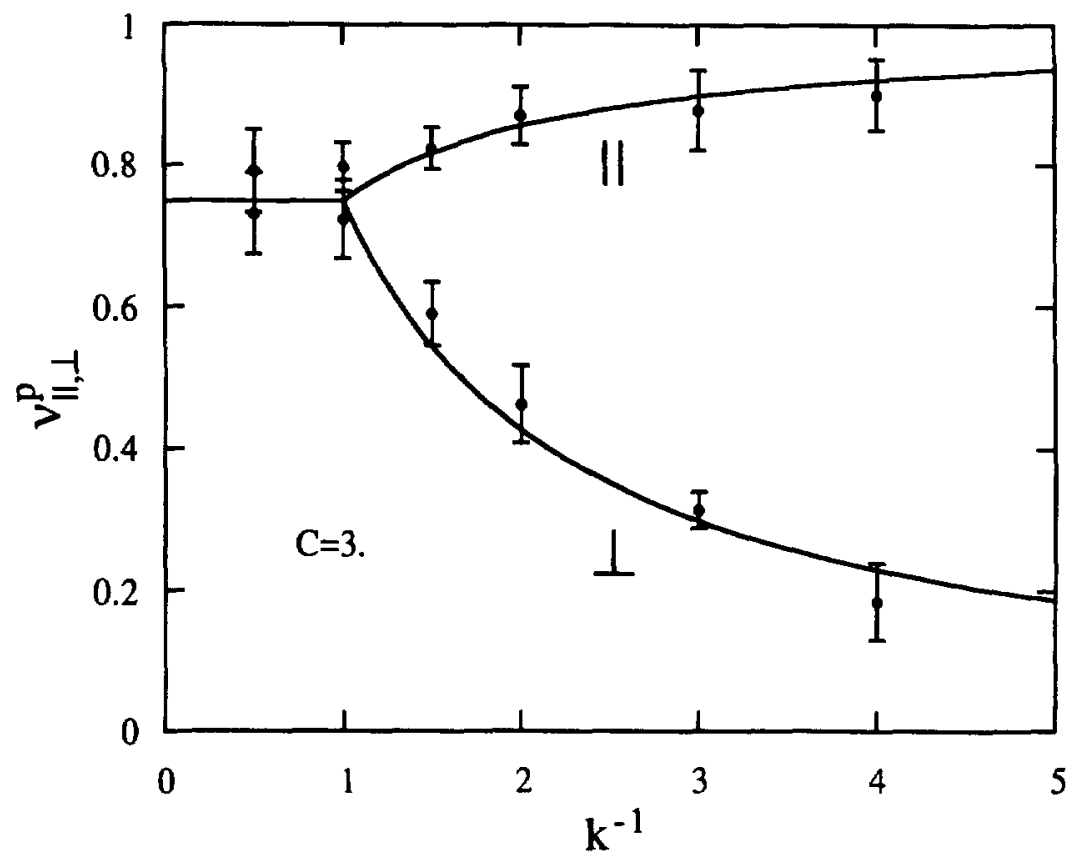

Fig. 5. - Variation of the radius exponents with $k^{-1}$ for $C=3$. The surface geometry is relevant and induces exponent anisotropy when $k<1$. 


\section{Blob picture approach and Flory approximation.}

Below the upper critical dimension when self-avoidance governs the chain configuration, a confined chain with $k<1$ may be described using the blob picture [10-11]. Inside a parabola in $2 \mathrm{~d}$ or a paraboloid in $3 \mathrm{~d}$, the chain configuration results from the piling up of isotropic fractal blobs as shown in figure 1 . Since $k<1$, the first blob has a radius $R_{C}$, which is also the typical length introduced by the surface as given by equation (5). The next blobs are tangent both to the surface and to the preceding one. Within the blobs the correlations are the same as for an unconstrained chain in $d$ dimensions and the number of monomers inside the $p$-th blob is related to its radius through

$$
n_{p} \sim r_{p}^{D}
$$

where $D$ is the fractal dimension given by $1 / \nu$. Let the blob center be located at $\left(u_{p}, 0\right)$, then according to (1)

$$
r_{p}=C u_{p}^{k}
$$

and

$$
u_{p+1}-u_{p}=r_{p}+r_{p+1} .
$$

For a long chain one may use a continuum approximation in $p$ writing the blob radius as

$$
r_{p} \sim C^{\ell} p^{m}
$$

Then from (12) and (13)

$$
\begin{aligned}
u_{p} & =C^{(\ell-1) / k} p^{m / k} \\
\frac{\partial u_{p}}{\partial p} & \simeq 2 r_{p} \sim C^{\ell} p^{m}
\end{aligned}
$$

Combining the last two equations allows a determination of the exponents $\ell=1 /(1-k)$ and $m=k /(1-k)$ and finally

$$
r_{p} \sim C^{1 /(1-k)} p^{k /(1-k)} \quad u_{p} \sim C^{1 /(1-k)} p^{1 /(1-k)}
$$

When the chain contains $P$ blobs and $N$ monomers, according to (11)

$$
N \sim \sum_{p=1}^{P} r_{p}^{D} \sim C^{D /(1-k)} \sum_{p=1}^{P} p^{D k /(1-k)} \sim C^{D /(1-k)} P^{[1+k(D-1)] /(1-k)}
$$

so that

$$
\begin{gathered}
R_{\|} \sim u_{P} \sim C^{(1-D) /[1+k(D-1)]} N^{1 /[1+k(D-1)]} \\
R_{\perp} \sim r_{P} \sim C^{1 /[1+k(D-1)]} N^{k /[1+k(D-1)]}
\end{gathered}
$$

From these expressions one can deduce the scaling function exponents defined in equation (7)

$$
\omega_{\|}=\frac{(k-1)(1-\nu)}{\nu+k(1-\nu)} \quad \omega_{\perp}=\frac{(1-k) \nu}{\nu+k(1-\nu)} .
$$

They satisfy the scaling relation (9) and give the asymptotic slopes given by the dashed lines in 
figure 3. The chain radius exponents can be identified as

$$
\nu p=\frac{1}{1+k(D-1)}=\frac{\nu}{k+\nu(1-k)} \quad \nu_{\perp}^{p}=k \nu p .
$$

When $k=1$ the perturbation is marginal and the radius exponents keep the flat surface value with $\nu P=\nu_{\perp}^{p}=\nu$. Otherwise when $k=0$ the chain is confined in a $1 \mathrm{~d}$ geometry with $\nu P=1$. In $2 \mathrm{~d}$ the Flory value $\nu=3 / 4$ is exact [17] and can be introduced in (22) to compare to the Monte-Carlo results. This is done in figure 5 where a reasonable agreement is obtained.

The Flory theory $[18-19,10]$ provides an alternative approach to the problem. Although approximate, it is known to give good estimates of the correlation length exponents and will allow us to discuss the upper critical dimension of the system.

A trial free energy is written as the sum of an elastic part of entropic origin, evaluated for an ideal chain, and a repulsive energy contribution obtained in a meanfield approximation. In $d$ dimensions, the volume occupied by the chain in the paraboloid is

$$
V\left(R_{\|}\right) \sim C^{d-1} R^{+k(d-1)}
$$

so that the free energy is given by

$$
F\left(R_{\|}\right) \simeq \frac{R^{2}}{N}+\frac{N^{2}}{C^{d-1} R_{\|}^{+k(d-1)}}
$$

where we ignored a transverse contribution to the entropy of order $R_{\|}^{2 k} / N$. The free energy is a function of the variable $R_{\|}$only since, due to the confinement, $R_{\|}$and $R_{\perp} \sim R_{\|}^{k}$ are not independent quantities. The free energy minimum corresponds to

$$
R_{\|} \sim C^{(1-d) /[3+k(d-1)]} N^{3 /[3+k(d-1)]}
$$

In the limit $k \rightarrow 1$ one recovers the Flory radius for a free chain with the fractal dimension

$$
D_{\mathbf{F}}=\frac{d+2}{3}
$$

It may be verified that (25) also follows from (19) when $D=D_{\mathrm{F}}$ is used in the blob result for $R_{\|}$.

The upper critical dimension for the free SAW is $d_{c}^{0}=4$ corresponding to the ideal chain fractal dimension $D=2$. For the confined chain, it will be given by $\nu P\left(d_{c}\right)=1 / 2$ so that, using the Flory expression, one gets

$$
d_{\mathrm{c}}=1+\frac{3}{k} \quad k \leqslant 1
$$

As usual for anisotropic systems, $d_{c}$ is higher than in the isotropic case. This result may also be deduced from the behaviour of the repulsive energy term in (24) [10]. Replacing $R_{\|}$by the ideal chain value $N^{1 / 2}<R_{\|}$, the repulsive part is at most of order $N^{[3-k(d-1)]^{2}}$ while the elastic energy is at least $O(1)$. It follows that the confined chain is ideal above $d_{\mathrm{c}}$ given in equation (27). Another way to reach the same conclusion is to consider that, according to equation (23), the volume of the system scales like $R_{\|}^{d_{\text {eff }}}$, with an effective dimension $d_{\text {eff }}(d)=1+k(d-1)$ which is equal to $d_{c}^{0}=4$ at the upper critical dimension given by (27). Finally, keeping $D=D_{\mathrm{F}}$ given by (26) between $d_{\mathrm{c}}^{0}$ and $d_{\mathrm{c}}$, the blob result still agrees with the Flory theory as if, due to the confinement, the blob fractal dimension were increased above the ideal chain value. 


\section{Discussion.}

An anisotropic critical system is left invariant under the anisotropic change of the length scales by $b^{z}$ in the parallel direction and $b$ in the transverse one, where $z$ is the anisotropy exponent given by the ratio $\nu_{\|} / \nu_{\perp}$. When this scaling transformation is applied to the surface geometry in (1), equation (2) becomes

$$
C^{\prime-1}=b^{1-z k} C^{-1}
$$

Thus the surface geometry is invariant and the system marginal for $z=k^{-1}$. This has already been verified for the directed walk, with $z=2$, confined inside a parabola with $k=1 / 2$ [2021].

For the SAW studied here, the original isotropic fixed point is unstable and, under the influence of the relevant surface perturbation, the system is driven towards a new anisotropic fixed point with an exponent ratio $z$ adjusting self-consistently to the marginal value $k^{-1}$ and leaving the geometry invariant, a necessary condition for the mere existence of a fixed point. It would be interesting to study the number of walks in order to obtain the «magnetic » exponents at the new fixed point. Simulations using the grand canonical ensemble would also be useful to determine the critical fugacity which has to be different from the bulk one since the conformal mapping of the half-space on the parabola leads to a finite correlation length at the bulk critical fugacity [9].

As an extension of the present work one may consider other polymer systems in the Flory approximation. Let us just mention some results :

- Polymer chain in a $\Theta$-solvent [22] :

$$
\nu p=\frac{2}{2+k(d-1)} \quad d_{c}=1+\frac{2}{k} .
$$

- Branched polymer in a good solvent [23-24]:

$$
\nu P=\frac{5}{6+2 k(d-1)} \quad d_{\mathrm{c}}=1+\frac{7}{k} .
$$

- Branched polymer in a $\Theta$-solvent [25]:

$$
\nu p=\frac{7}{8+4 k(d-1)} \quad d_{\mathrm{c}}=1+\frac{5}{k} .
$$

The blob method has already been used to study fractal growth process on a strip [26]. This may be extended to the case of fractal growth in parabolic geometry. The knowledge of the fractal dimension $D$ for free growth immediately gives $\nu p$ when inserted into equation (22).

Finally this approach can be generalized to study systems displaying anisotropic critical behaviour from the start with $\nu_{\|} / \nu_{\perp}=z$. Then, one has to consider anisotropic blobs with a transverse radius $r_{p \perp}$, a length $r_{p \|}$, containing $n_{p} \sim r_{p \perp}^{1 / \nu_{\perp}} \sim r_{p \|}^{1 / \nu_{1}}$ monomers and piling up in the parabolic geometry. A simple calculation gives

$$
\nu p=\frac{1}{1+z k\left(1 / \nu_{\|}-1\right)} \quad \nu_{\perp}^{p}=k \nu p \quad k \leqslant z^{-1}
$$

Both exponents are left unchanged in the marginal case $k=z^{-1}$. The parallel exponent also keeps its original value for any $k$ if $\nu_{\|}=1$, a situation encountered with the directed walk $[20,21]$. 


\section{Acknowledgments.}

Discussions with Ingo Peschel and Ferenc Igloi at an early stage of this work are gratefully acknowledged.

\section{References}

[1] Cardy J. L., J. Phys. A 16 (1983) 3617.

[2] Cardy J. L., Nucl. Phys. B 240 [FS12] (1984) 514.

[3] Cardy J. L., Redner S., J. Phys. A 17 (1984) L933.

[4] Barber M. N., Peschel I., Pearce P. A., J. Stat. Phys. 37 (1984) 497.

[5] Peschel I., Phys. Lett. 110A (1985) 313.

[6] Kaiser C., Peschel I., J. Stat. Phys. 54 (1989) 567.

[7] Davies B., Peschel I., J. Phys. A 24 (1991) 1293.

[8] Guttmann A. J., Torrie G. M., J. Phys. A 17 (1984) 3539.

[9] Peschel I., Turban L., Igloi F., J. Phys. A 24 (1991) L1229.

[10] De Gennes P.-G., Scaling Concepts in Polymer Physics (Cornell University Press, Ithaca, N.Y., 1979) chap. I.

[11] Daoud M., Cotton J. P., Farnoux B., Janninck G., Sarma G., Benoit H., Duplessix R., PICOT C., DE GENNES P. G., Macromolecules 8 (1975) 804.

[12] Kremer K., Binder K., Computer Phys. Rep. 7 (1988) 259.

[13] Motroll E. W., J. Chem. Phys. 18 (1950) 734.

[14] Lal M., Molec. Phys. 17 (1969) 57.

[15] Wall F. T., ERpenbeck J. J., J. Chem. Phys. 30 (1959) 634.

[16] Madras N., Sokal A. D., J. Stat. Phys. 50 (1988) 109.

[17] Nienhuis B., Phys. Rev. Lett. 49 (1982) 1062.

[18] FlORY P., Principles of Polymer Chemistry (Cornell University Press, Ithaca, N.Y., 1971) chap. XII.

[19] Fisher M. E., J. Phys. Soc. Japan Suppl. 26 (1969) 44.

[20] Turban L., J. Phys. A 25 (1992) L127.

[21] IgLoI F., Phys. Rev. A 45 (1992) 7024.

[22] Raphael E., Pincus P., J. Phys. II France 2 (1992) 1341.

[23] IsaAcson J., Lubensky T. C., J. Phys. Lett. France 41 (1980) L469.

[24] Daoud M., Joanny J. F., J. Phys. France 42 (1981) 1359.

[25] DaOud M., Pincus P., StockmaYeR W. H., Witten Jr T., Macromolecules 16 (1983) 1833.

[26] Turban L., Debierre J. M., J. Phys. A 17 (1984) L289. 